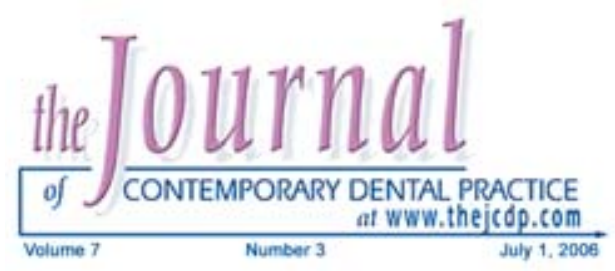

\title{
Clinical Evaluation of Post-Extraction Site Wound Healing
}

\section{Wasiu Lanre Adeyemo, BDS, FMCDS, Dr.Med.Dent.; \\ Akinola Ladipo Ladeinde, BDS, FMCDS, FWACS; \\ Mobolanle Olugbemiga Ogunlewe, BDS, FWACS}

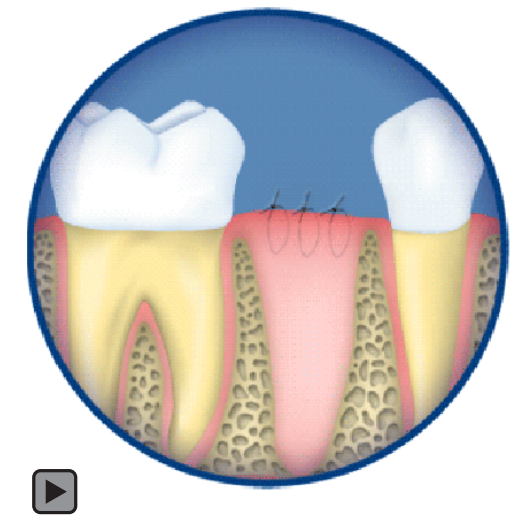

Abstract

Aim: The aim of this prospective study was to evaluate the clinical pattern of post-extraction wound healing with a view to identify the types, incidence, and pattern of healing complications following non-surgical tooth extraction.

Study Design: A total of 311 patients, who were referred for non-surgical (intra-alveolar) extractions, were included in the study. The relevant pre-operative information recorded for each patient included age and gender of the patient, indications for extraction, and tooth/teeth removed. Extractions were performed under local anesthesia with dental forceps, elevators, or both. Patients were evaluated on the third and seventh postoperative days for alveolus healing assessment. Data recorded were: biodata, day of presentation for alveolus healing assessment, day of onset of any symptoms, body temperature $\left({ }^{\circ} \mathrm{C}\right)$ in cases of alveolus infection, and presence or absence of pain.

Results: Two hundred eighty-two patients (282) with 318 extraction sites were evaluated for alveolus healing. Healing was uneventful in 283 alveoli (89\%), while 35 alveoli (11\%) developed healing complications. These complications were: localized osteitis $26(8.2 \%)$; acutely infected alveolus $5(1.6 \%)$; and an acutely inflamed alveolus $4(1.2 \%)$. Females developed more complications than males $(p=0.003)$. Most complications were found in molars $(60 \%)$ and premolars $(37.1 \%)$. Localized osteitis caused severe pain in all cases, while infected and inflamed alveolus caused mild or no pain. Thirty patients (12\%) among those without healing complications experienced mild pain.

(c) Seer Publishing 
Conclusions: Most of the post-extraction alveoli healed uneventfully. Apart from alveolar osteitis (AO), post-extraction alveolus healing was also complicated by acutely infected alveoli and acutely inflamed alveoli. This study also demonstrated a painful alveolus is not necessarily a disturbance of post-extraction site wound healing; a thorough clinical examination must, therefore, be made to exclude any of the complications.

Keywords: Evaluation, post-extraction alveolus, alveoli, healing

Citation: Adeyemo WL, Ladeinde AL, Ogunlewe MO. Clinical Evaluation of Post-extraction Site Wound Healing. J Contemp Dent Pract 2006 July;(7)3:040-049.

\section{Introduction}

Extraction of teeth is the most common procedure carried out in oral surgery clinics. Uneventful healing of a post-extraction alveolus occurs in most cases following dental extraction. ${ }^{1,2}$ However, occasionally healing is complicated even in normal healthy patients. ${ }^{2}$ Few extraction wounds, estimated at 1.0 to $11.5 \%$, have been reported to heal improperly or incompletely. ${ }^{1,3-11}$ Although, the incidence of complications with post-extraction alveolus healing is minor, the problems created by the disturbances in post-extraction wound healing are not only limited to localized symptoms (pain, exudate discharge, foul odor), but lost days at work and decreased productivity from frequent hospital visits. Disturbed healing can also complicate or even jeopardize dental implant placement and other procedures. ${ }^{12}$ Three different types of complications of healing of post-extraction alveolus are recognized: alveolar osteitis (AO), acutely infected alveolus, and acutely inflamed alveolus. ${ }^{3-11}$ Of all the complications, $\mathrm{AO}$ is the most common and most widely discussed in the literature and is sometimes confused with other less common complications. ${ }^{13,14}$

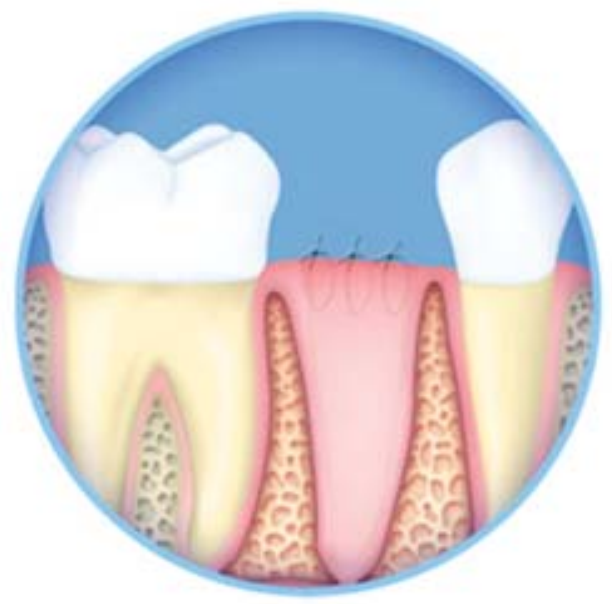

Pain is a natural bodily response to noxious stimuli. In post-extraction wound healing pain is a key factor alerting patients to seek professional care out of concern for disturbed healing. ${ }^{9}$ Normal uncomplicated alveolus healing has also been reported to cause moderate to severe pain. ${ }^{9}$

The aim of this study was to evaluate the clinical pattern of post-extraction wound healing with the intention of identifying the types, incidence, and pattern of healing complications following nonsurgical tooth extraction.

\section{Methods and Materials}

For this study, 40 caries-free human first This prospective study was conducted at the Exodontia Clinic of the Department of Oral and Maxillofacial Surgery at the Lagos University Teaching Hospital (LUTH) in Lagos, Nigeria from October 2002 to January 2003. The study had the approval of the Research and Ethics Committee of the hospital. Subjects selected were those referred for nonsurgical tooth extractions. Informed consent was obtained from each subject in the study. The relevant pre-operative information recorded for each patient included age and gender of the patient, indications for extraction (the diagnosis was based on both clinical and radiographic examinations), and tooth/teeth removed.

The following groups of patients were excluded from the study: patients who were taking antibiotics for an existing infection; those with underlying medical conditions such as diabetes mellitus, severe nutritional deficiencies, endocrine disturbances; those with social habits such as cigarette smoking and alcohol consumption; patients on oral contraceptives and steroid therapy; and those with a history of radiotherapy for the treatment of head and neck malignancies. 


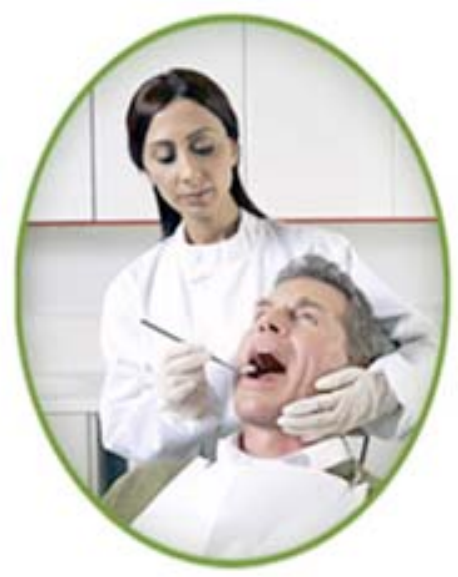

Extractions were performed under local anaesthesia ( $2 \%$ xylocaine with epinephrine $1: 80,000)$ with dental forceps, elevators, or both. No postoperative antibiotics were prescribed. Paracetamol (1 gm 2 hours postoperatively and then 1 gm every 8 hours for 24 hours) was prescribed for all patients. Identical postextraction instructions were given to all the patients, and all were evaluated for alveolus healing on the third and seventh days following surgery. They were also instructed to report to the clinic if there was any increased or persistent pain in the post-extraction alveolus within seven days following the extraction or beyond.

An alveolus healing assessment form was completed for each patient, and this included the patient's biodata, day of presentation for alveolus healing assessment, day of onset of any symptoms, body temperature $\left({ }^{\circ} \mathrm{C}\right)$ in cases of alveolus infection, and presence or absence of pain.

Each patient was asked about the intensity of pain experienced in each individual alveolus throughout the postoperative period (seven days). For the purpose of this study, a four-point verbal rating scale (VRS) questionnaire was used: 'no pain' (patient experiences no pain); 'mild pain' (pain almost unnoticeable); 'moderate pain' (noticeable pain but patient can still engage in routine daily activities); 'severe pain' (pain very noticeable and disturbs patient's daily routine). This was recorded for each patient in the questionnaire. The recordings were analyzed on a scale of 0-3 (0-no pain, 1-mild pain, 2-moderate pain, 3-severe pain).
The clinical evaluation of the post-extraction alveolus healing was based on the following criteria:
Alveolar Osteitis: Persistent or increased post-operative pain in and around the extraction site not adequately relieved by mild analgesics. The pain was accompanied by a partially or totally disintegrated blood clot or an empty alveolus with or without the presence of halitosis. The diagnosis is confirmed when extremely sensitive bare bone is encountered by inserting a small curette into the extraction wound.

Acutely Inflamed Alveolus: A painful alveolus with profoundly inflamed tissue but without exudate or an elevated body temperature.

Acutely Infected Alveolus: A painful alveolus with suppuration, erythema, and edema with or without systemic fever.

Normal Healing Alveolus: An alveolus with normal granulation tissue with or without pain.

\section{Statistical Analysis \\ Data was analyzed using the SPSS for Windows (version 11.5; SPSS Inc, Chicago, IL, USA) statistical software package. Descriptive statistics and Chi-square test $\left(X^{2}\right)$ for independence between categorical variables were used as appropriate. The critical level of significance was set at $\mathrm{P}<.05$.}

\section{Results}

A total of 347 teeth were extracted from 311 patients who participated in the study. The mean age (SD) of patients was $34.60 \pm 15.95$ years (range, 11-72 years). Thirty-five percent (109) of patients who constituted the majority were in age group 21-30 years (Figure 1).

Male to female ratio was 1:1.5 (125 males and 186 females). Dental caries and its sequelae, i.e., pulpitis, apical periodontitis, and retained roots was the most common indication for dental extraction accounting for $62.3 \%$ (216 teeth) 


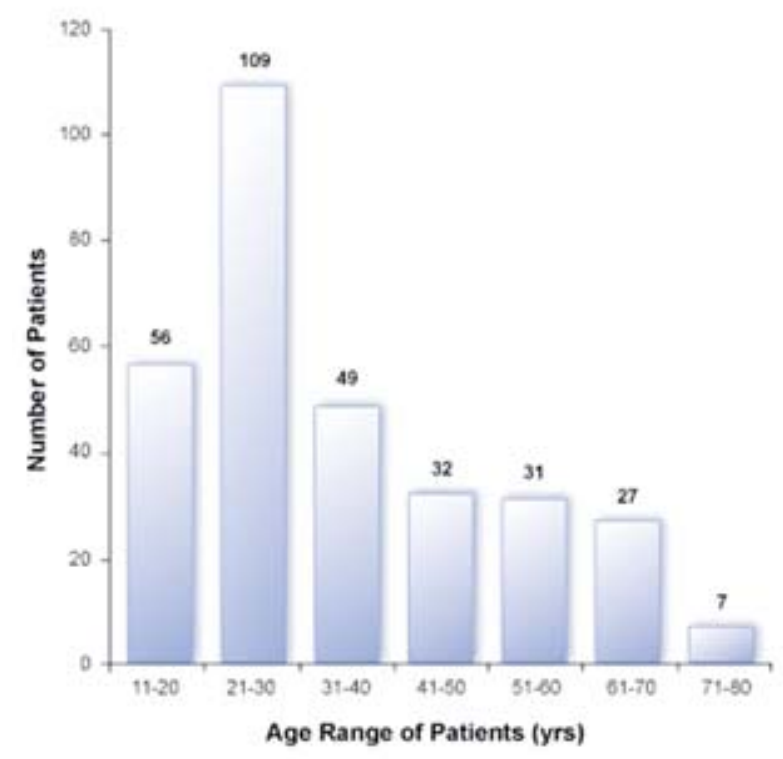

Figure 1. Age range of participants in the study.

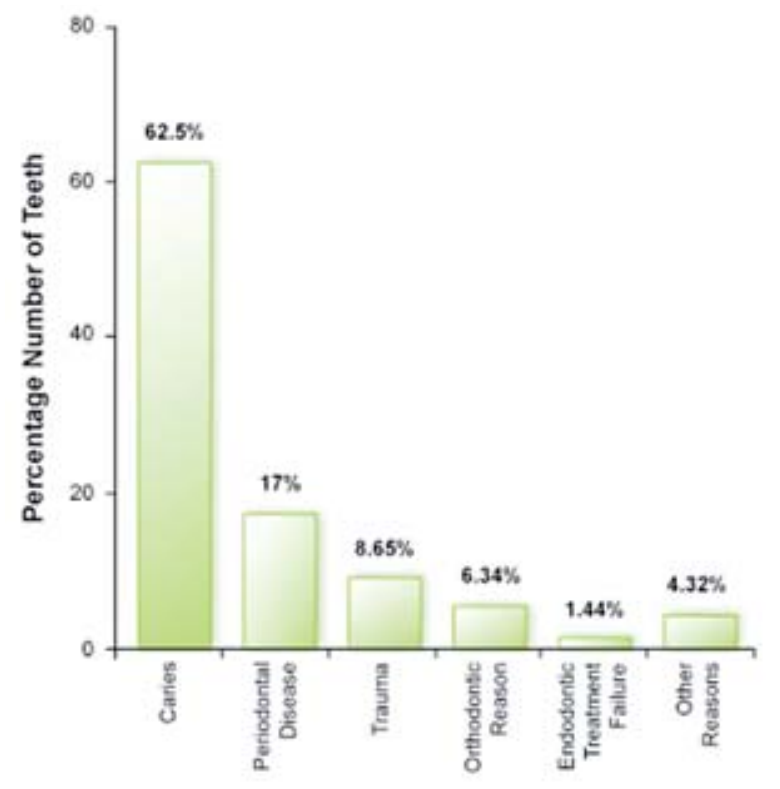

Figure 2. Indications for extraction.

followed by periodontal diseases 17\% (59 teeth) (Figure 2).

Two hundred eighty-two patients (282) with 318 extraction sites were assessed for socket healing. Healing was uneventful in 250 patients with 283 extraction sites (89\%), while healing complications developed in 32 patients with 35 extraction sites (11\%). The complications were: $26(8.2 \%)$ with dry socket; acutely infected alveoli 5 (1.6\%); and acutely inflamed alveoli 4 (1.2\%) (Figure 3).

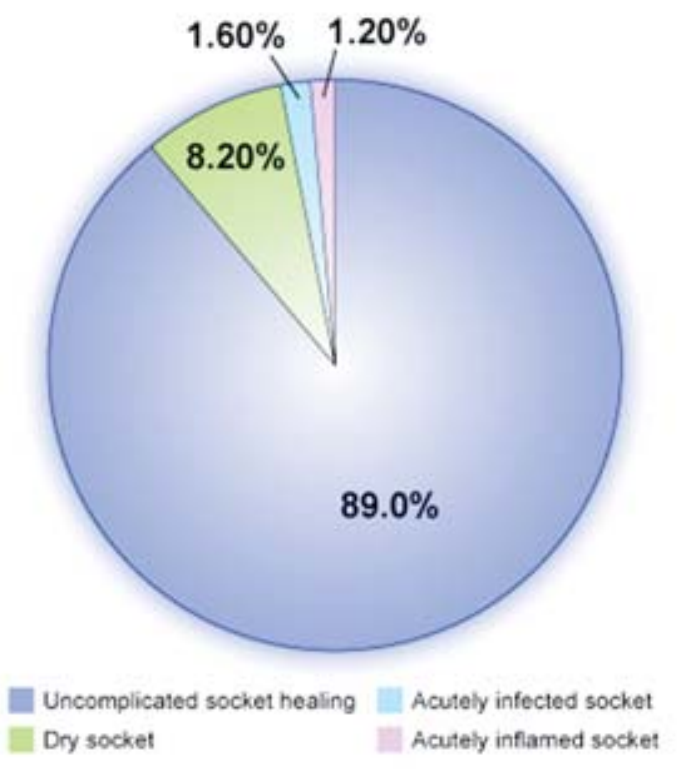

Figure 3. Healing status in 318 extraction alveoli.

Dry socket (alveolar osteitis) was the most common of the alveolus healing complications constituting $74.3 \%$ (26 alveoli), while acutely infected alveoli and acutely inflamed alveoli constituted $14.3 \%$ (5 alveoli) and $11.4 \%$ (4 alveoli), respectively.

Patients with post extraction alveolus complications were between 13-57 years of age with mean age (SD) of $30.59 \pm 13.1$. Females (27) developed more complications than males (5) $\left(p=0.003, x^{2}=8.98\right)$ (Table 1). Alveolar healing complications were observed in molars $(60 \%)$, premolars $(37.1 \%)$, and incisors (2.9\%). Eighteen maxillary and 17 mandibular teeth (ratio 1:1.05) were involved. Table 2 shows the alveolar healing complications and types of teeth involved.

Normal Healing Alveoli: Two hundred fifty patients had uneventful healing. By the third day following extraction, $220(88 \%)$ of them experienced no more pain. Twenty-four (9.6\%) patients experienced mild (VRS level 1) or moderate pain (VRS level 2) up until day three, while six patients $(2.4 \%)$ continued to experience mild pain (VRS level 1) throughout the seven day postoperative period.

Alveolar Osteitis: Twenty-six alveoli in 23 patients developed this complication. The age range of patients was 13-57 years with the 
Table 1. Gender of patients and alveolus healing status.

\begin{tabular}{|l|c|c|c|}
\hline \multicolumn{1}{|c|}{ Postoperative Healling of Alveoli } \\
\cline { 2 - 4 } SEX & $\begin{array}{c}\text { Normal healing } \\
\text { alveolus }\end{array}$ & $\begin{array}{c}\text { Complicated healing } \\
\text { alveolus }\end{array}$ & Total \\
\hline Male & 108 & 5 & 113 \\
\hline Female & 142 & 27 & 169 \\
\hline Total & 250 & 32 & 282 \\
\hline
\end{tabular}

$\left(x^{2}=8.98 ; p=0.003\right)$

Table 2. Socket healing complications and types of teeth involved.

\begin{tabular}{|l|c|c|c|c|}
\hline \multicolumn{5}{|c|}{ Socket Healing Complications } \\
\cline { 2 - 5 } Types of teeth & Dry Socket & Inflamed socket & $\begin{array}{r}\text { Infected } \\
\text { socket }\end{array}$ & Total (\%) \\
\hline Molars & 19 & 0 & 2 & $21(60)$ \\
\hline Premolars & 7 & 4 & 2 & $13(37.1)$ \\
\hline Incisors & 0 & 0 & 1 & $1(2.9)$ \\
\hline Total & 26 & 4 & 5 & $35(100)$ \\
\hline
\end{tabular}

majority $(30.4 \%)$ found in age group of $21-30$ years. The male:female ratio was 1:4.8 All the patients presented between two to five days after the extraction with severe, deep-seated pain in and around the extraction site (VRS level 3). Fourteen patients $(60.9 \%)$ presented with a greyish soft friable mass (disintegrated clot) in their alveoli, while the remaining nine patients presented with empty alveoli with bone exposure. As a group, the third molars (34.6\%) were most frequently affected, followed by the first molars $(23.1 \%)$, second molars $(15.4 \%)$, first premolars (15.4\%), and second premolars $(11.5 \%)$. The tooth most frequently affected was the mandibular third molar (23.1\%).

The treatment protocol included minimal alveolus curettage with irrigation of the alveolus using warm normal saline, done every other day until the symptoms subsided and granulation tissue was well established in the alveolus. All of these patients were given pain medication (tablets Ibuprofen $400 \mathrm{mg}$ ) every 12 hours for three days.
The duration of symptoms after commencement of treatment ranged from two to four days and healing was subsequently uneventful.

Acutely Infected Alveolus: All five patients (4 females; 1 male) with this complication presented on the third day following the extraction with discharge of exudate and minimal swelling of the soft tissue surrounding the alveolus. No patients presented with elevated body temperatures. Four patients presented with mild pain (VRS level 0-1), and one patient had no pain at presentation. The treatment protocol for these patients included irrigation of the alveolus with warm normal saline and oral administration of Ampiclox (500 mg every 6 hours daily for five days) and metronidazole (400 mg every 8 hours daily for five days). Healing was subsequently uneventful.

Acutely Inflamed Alveolus: Two patients in this group presented with profound erythematous inflamed tissue in their alveoli 20 days after the 
extraction, while the other two patients presented 24 days after the extraction. All the patients were females, and they all presented with mild pain or no pain at all (VRS level 0-1). One of the patients had a positive history of hypertrophic scar/keloid formation. The treatment protocol for these patients consisted of curettage of the inflammed tissue from the alveolus followed by pressure control of bleeding. Neither antibiotics nor analgesics were prescribed. Healing was subsequently uneventful.

\section{Discussion}

A post-extraction alveolus heals normally in five overlapping stages, which involves clotting, replacement of blood clot with healthy granulation tissue, gradual replacement of granulation tissue by connective tissue and young preosseous tissue, and by the thirty-eighth day bone trabeculae fills at least two-thirds of the alveolus. ${ }^{12,15-17}$ Closure of epithelium begins as regeneration on the fourth day, with complete fusion in closure of the alveolus after 24-35 days. Knowledge of these stages is helpful to identify the pathogenesis of healing disturbances.

In a histological study, Amler ${ }^{12}$ reported $\mathrm{AO}$ results from disturbances in the progression of healing from blood clot to granulation tissue. Failure or interference in the mechanism of the granulation tissue development to replace the clots results in disintegration of the blood clot by putrefaction rather than by orderly resorption, giving rise to the well-known symptoms of dry socket. He also reported histologic examination of infected alveoli usually reveals a breakdown of the granulation tissue with an influx of pyogenic cells usually with a preponderance of neutrophils and a few plasma cells and monocytes. ${ }^{12}$ No significant organization of healing is evident. Clinical presentation of an acutely inflamed alveolus in the present series suggested a disturbance during the third stage of healing with exuberant inflamed tissue over-filling the alveolus.

The majority of the extraction sites (89\%) in this study had uneventful healing, which is in agreement with other authors. . $^{3,6,8,9-11}$ The mean age of patients, onset of symptoms, type and site of teeth affected, as well as gender of patients affected by socket healing complications are also similar to previous studies. ${ }^{6,718}$ Posterior teeth (molars and premolars) constituted the majority of teeth affected by socket healing complications in this study. This is consistent with many reports in the literature. ${ }^{6,7,19}$ Mandibular and maxillary teeth were affected by socket healing complications almost equally in this study in contrast to most reports in the literature,,$^{5,11,20,21}$, which reported mandibular teeth are more affected than maxillary teeth.

In the present study three different types of post-extraction site wound healing complications were identified: $\mathrm{AO}$, the acutely infected alveolus, and the acutely inflamed alveolus. Of all these complications, $\mathrm{AO}$ is the most widely discussed in the literature as the most common complication of post-extraction site wound healing. ${ }^{1,-11}$ Cheung et al. ${ }^{9}$ also identified three different types of extraction site wound healing disturbances in their study. Cheung et al. ${ }^{9}$, however, reported acutely inflamed alveoli as the most frequently encountered complication of post-extraction site wound healing in contrast to our findings and other established reports. ${ }^{1,5,7,9,11,19-}$

${ }^{21}$ Few other studies have also reported infected alveoli in addition to $\mathrm{AO}$ as a complication of post-extraction site wound healing. ${ }^{8-10}$

AO constituted $74.3 \%$ of healing complications in the present study. This confirms the long held belief $A O$ is the most frequently encountered healing complication following extraction of permanent teeth in humans. The peak age incidence (21-30 years) of AO in this study is similar to findings reported by Oginni et al. ${ }^{11}$ and Amaratunga and Senaratne ${ }^{7}$ but is at variance with the report of MacGregor. ${ }^{22}$ The age range of 13 to 57 years found in the present study is, however, similar to Oginni et al. ${ }^{11}$ and Macgregor. ${ }^{22}$ This is contrary to the claim AO seldom or never occurs before the eighteenth or after the fiftieth year of life. ${ }^{23}$ The onset of symptoms, commonly affected teeth (posterior versus anterior), and other symptoms of $\mathrm{AO}$ are comparable to other reports. ${ }^{2,711}$ The third molars were the most commonly affected in this study followed by the first molars and then second molars. This is in contrast with the first molars, second molars, and third molars in descending order reported by others. ${ }^{4,11}$ 
Acutely infected alveoli constituted $14.3 \%$ of healing complications in this study, with an incidence of $1.6 \%$. This is in contrast with earlier reports in the literature. ${ }^{8-10}$ Cheung et al. ${ }^{9}$ reported an incidence of $0.5 \%$, while Simon and Matee ${ }^{8}$ reported infected alveoli constituted $48.7 \%$ of all post-extraction site healing complications in their study. Cheung et al. ${ }^{9}$ reported systemic fever in all their patients with acutely infected alveoli, whereas patients with acutely infected alveoli in the present study did not have an associated elevation of body temperature. Acutely infected alveoli were also considered a local infection without any associated fever in another report. ${ }^{8}$

Although the acutely inflamed alveolus was first described by Cheung et al. ${ }^{9}$, a lesion of similar clinical characteristics was earlier reported by Leong and Seng. ${ }^{24}$ An acutely inflamed alveolus constituted $88.4 \%$ of post-extraction site healing complications, with an incidence of $11.1 \%$ in the report by Cheung et al. ${ }^{9}$; whereas acutely inflamed alveoli in the present study constituted $11.4 \%$ of the post-extraction site healing complications with an incidence of $1.2 \%$. Patients with inflamed alveoli in this study presented between 20-24 days after the extraction in contrast to the other report, ${ }^{9}$ where the patients presented within seven days following the extraction.

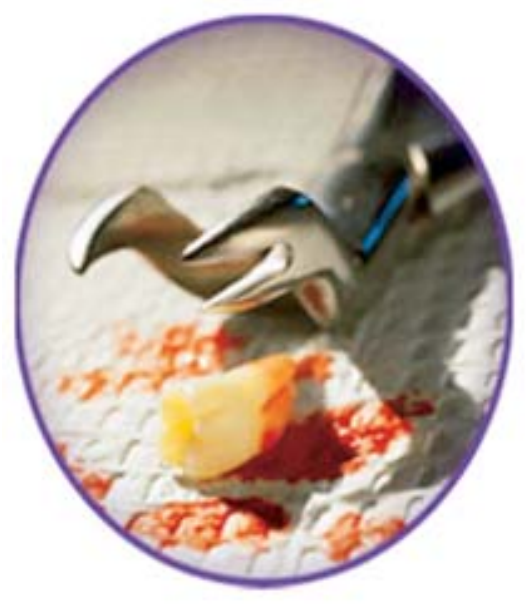

Interestingly, one patient with a positive history of hypertrophic scar presented with an acutely inflamed alveolus. Hypertrophic scars and keloids represent genetic pathologic "overhealing" states. $^{25}$ These lesions are caused by excessive production and deposition of collagen and glycoprotein without equivalent degradation. The relationship between an inflamed alveolus and hypertrophic scarring requires further investigation. The possibility of a genetic association with an inflamed alveolus is also worthy of further investigation.

Female patients were significantly affected by socket healing complications in this study (maleto-female ratio of 1:5.4). A significantly higher incidence of socket healing complications, especially $\mathrm{AO}$ in females, has been attributed to the use of oral contraceptives. ${ }^{2}$ Interestingly, none of the patients in this study admitted to being on oral contraceptives. Despite the argument of some authors to the contrary, many still support the belief female patients are slightly more prone to developing $\mathrm{AO}$ than males, even if the female patients are not taking oral contraceptives. ${ }^{26}$ Oginni et al. ${ }^{11}$ have also attributed this gender predilection to a better health-seeking behavior of females resulting in a higher rate of diagnosis of the condition than males. Since pain is an important symptom in the diagnosis of $A O$, some authors have postulated more females tend to have $A O$ because of their low pain threshold. ${ }^{14}$ The low pain threshold and better health-seeking behavior of females may also be responsible for the higher incidence of socket healing complications in females found in this study.

Pain is known to be a natural bodily response to noxious stimuli. ${ }^{9}$ In post-extraction wound healing pain is a key factor alerting patients to seek professional assessment because of concern for disturbed healing. In the present study, which agrees with Cheung et al. ${ }^{9}$, AO mostly caused severe pain, whereas the acutely infected alveolus was complicated mostly by moderate pain, and the acutely inflamed alveolus caused moderate or mild pain. In this study all the patients with $\mathrm{AO}$ presented with severe pain, whereas infected alveoli and inflamed alveoli caused mild or no pain at all. Normal uncomplicated socket healing was, however, associated with mild or moderate pain, up to the third day after the extraction in $9.6 \%$ of cases, while $2.4 \%$ of patients had mild pain throughout the seven day postoperative review. Although none of the patients with uncomplicated cases experienced severe pain, Cheung et al. ${ }^{9}$ reported 
about $17 \%$ of their patients with uncomplicated socket healing had moderate to severe pain at the seventh day recall.

The absence of absolute and objective clinical criteria and varying study designs have led to the confusion of socket healing complications with one another and with abnormal healing alveoli. ${ }^{13,14}$ Even though pain is a common symptom to these complications, $\mathrm{AO}$, which is the most frequently encountered complication, should not be confused with other complications as they have distinct clinical presentations.

\section{Conclusions}

In this study most of the extraction sites healed uneventfully. Apart from AO, post-extraction socket healing was also complicated by acutely infected alveoli and acutely inflamed alveoli. These complications were associated with distinct clinical signs and symptoms and different levels of pain experience. This study also demonstrated a painful alveolus is not necessarily a disturbance of post-extraction site wound healing. Therefore, a thorough clinical examination must be conducted to exclude any other complications.

\section{References}

1. Shafer WG, Hine MK, Levy BM. A Textbook of oral pathology. Philadelphia: W.B. Saunders Company; 1983. p. 601-5.

2. Seward GR, Harris M, McGowan DA. Killey and Kay's Outline of oral surgery Part 1. Bristol: IOP Publishing Ltd; 1987. p. 174-8.

3. Giglio JA, Rowland RW, Laskin DM, Grenevicki L, Roland RW. The use of sterile versus non-sterile gloves during out-patient exodontia. Quintessence Int 1993;24:543-4.

4. Oluseye SB. Exodontia: A retrospective study of the reasons, methods and complications of tooth extraction in oral and maxillofacial surgery clinic, Lagos University Teaching Hospital. NPMC dissertation. National postgraduate medical college of Nigeria. May, 1993.

5. Heasman PA, Jacobs DJ. A clinical investigation into the incidence of dry alveolus . Br J Oral Maxillofac Surg 1984;22:115-22.

6. Wagaiyu EG, Kaimenyi JT. Frequency of alveolar osteitis (dry alveolus ) at Kenyatta National Hospital Dental outpatient Clinic - a retrospective study. East Afr Med J 1989;66:658-62.

7. Amaratunga NA, Senaratne CM. A clinical study of dry alveolus in Sri Lanka. Int $\mathrm{J}$ Oral Maxillofac Surg 1988;26:410-18.

8. Simon E, Matee M. Post-extraction complications seen at a referral dental clinic in Dar Es Salaam, Tanzania. Int Dent J 2001;51:273-6.

9. Cheung LK, Chowe LK, Tsang MH, Tung LK. An evaluation of complications following dental extractions using either sterile or clean gloves. Int J Oral Maxillofac Surg 2001;30:550-4.

10. Jaafar N, Nor GM. The prevalence of post extraction complications in an out patient dental clinic in Kuala Lumpur Malaysia a retrospective survey. Singapore Dent J 2000;23: 24-8.

11. Oginni FO, Fatusi OA, Alagbe AO. A clinical investigation of dry alveolus in a Nigerian teaching hospital. J Oral Maxillofac Surg 2003;61:871-6.

12. Amler MH. Disturbed healing of extraction wounds J Oral Implant 1999;25:179-84.

13. Akota I, Alvsaker B, Bjornland T. The effect of locally applied gauze drain impregnated with chlortetracycline ointment in mandibular third molar surgery. Acta Odontolol Scan 1998;56:25-9.

14. Blum IR. Contemporary views on dry alveolus (alveolar osteitis): a clinical appraisal of standardization, aetiopathogenesis and management: a critical review. Int J Oral Maxillofac Surg 2002;31:309-17.

15. Amler MH, Johnson PL, Salman I. Histological and histochemical investigations of human alveolar alveolus healing in undisturbed extraction wounds. J Am Dent Assoc 1960;61:32-44.

16. Amler MH. Lag phase factor in bone healing and suggested application to marrow grafting. J Periodontal Res 1981;16:617-27.

17. Amler MH. The age factor in human alveolar bone repair. J Oral Implant 1993;19:138-42.

18. Cohen ME, Simecek JW. Effects of gender -related factors on the incidence of localized alveolar osteitis. Oral Surg Oral Med Oral Pathol Oral Radiol Endod 1995;79:416-22. 
19. Turner PS. A clinical study of "dry alveolus ". Int J Oral Surg 1982;11:226-31.

20. Rood JP, Murgatroyd J. Metronidazole in the prevention of dry alveolus . Br J Oral Surg 1979;17: 62-70.

21. Brown LR, Merrill SS, Allen RE. Microbiology study of intraoral wound. J Oral Surg 1970;28:89-95.

22. MacGregor AJ. Aetiology of dry alveolus : A clinical investigation. Br J Oral Maxillofac Surg 1968;6:49- 58.

23. Hermesch CB, Hilton TJ, Biesbrock AR, Baker RA, Gerlach RW, McClanahan SF. Perioperative use of $0.12 \%$ chlorhexidine gluconate for the prevention of alveolar osteitis. Oral Surg Oral Med Oral Pathol Oral Radiol Endod 1998;85:381-387.

24. Leong R. Seng GF. Epulis Granulomatosa: extraction sequellae. Gen Dent 1998;46 :252-5.

25. Papel ID. Facial plastic and reconstructive surgery. New York: Thieme Medical Publishers; 2002. p. 21.

26. Alexander RE. Dental extraction wound management: A case against medicating postextraction alveolus s. J Oral Maxillofac Surg 2000;58:538-51.

\section{About the Authors}
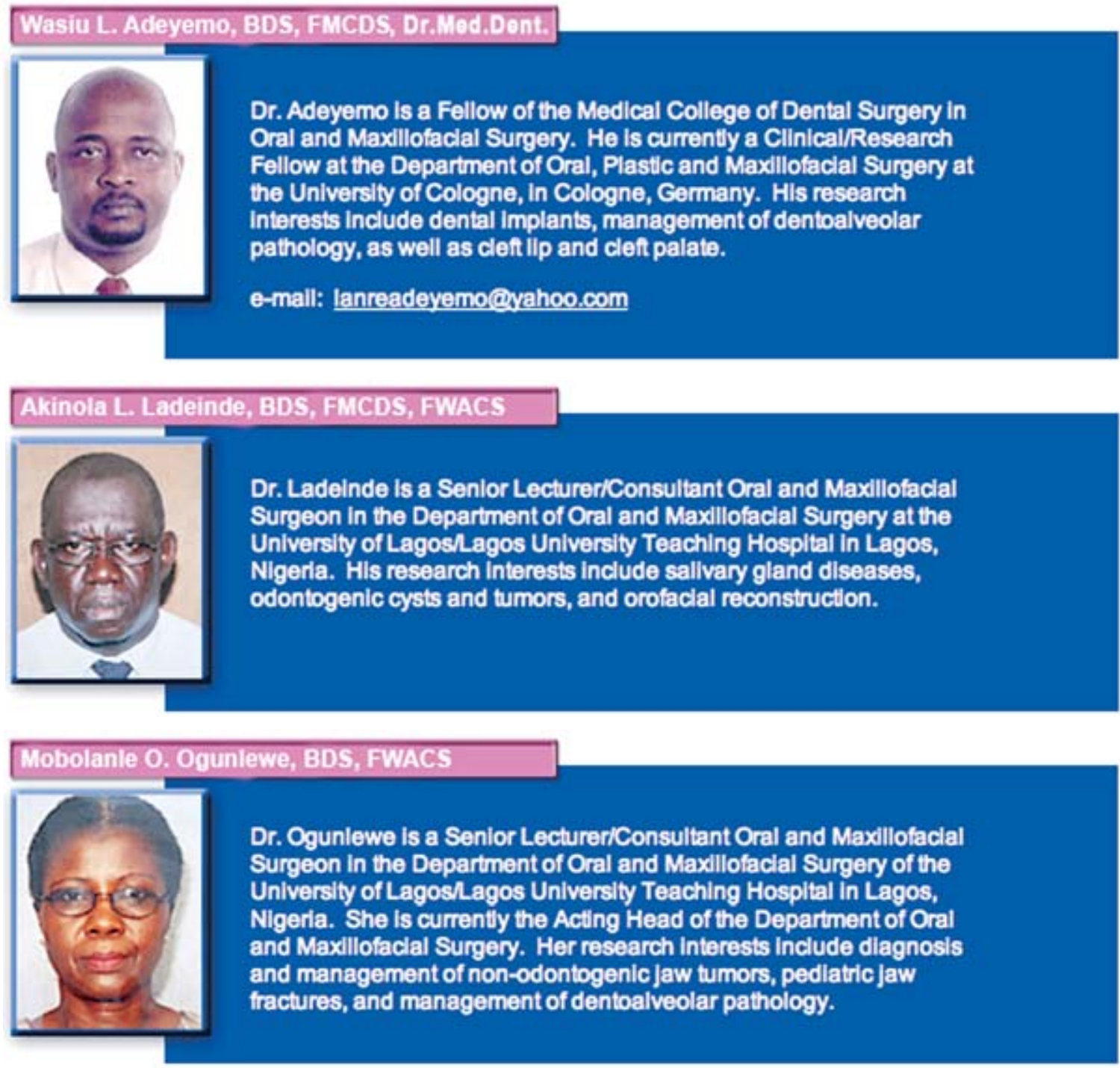